\title{
Propagation of Spreading Depression: A Review of Different Hypothesis
}

Mahmoud Lotfinia ${ }^{1,2 *}$, Ahmad Ali Lotfinia ${ }^{1}$, Babak Khodaie $^{1}$, Milad Ahmadi ${ }^{1}$, Sina Asaadi $^{3}$, Maryam Jafarian $^{1,4}$

${ }^{1}$ Shefa Neuroscience Research Center, Khatam Alanbia Hospital, Tehran, Iran.

${ }^{2}$ School of Medicine, Shahid Beheshti University of Medical Sciences, Tehran, Iran.

${ }^{3}$ Functional Neurosurgery Research Center, Shahid Beheshti University of Medical Sciences, Tehran, Iran.

${ }^{4}$ School of Advanced Technologies in Medicine, Tehran University of Medical Sciences, Tehran, Iran.

\section{A BSTRACT}

Introduction: Spreading depression is a transient and self-propagating wave of neuronal and glial depolarization, followed by a temporary loss of brain activities. Spreading depression is known by a huge redistribution of ions between extra- and intracellular spaces and spreads at the velocity of $2-3 \mathrm{~mm} / \mathrm{min}$ in all directions. Investigations indicate the role of spreading depression in several neurological disorders, including migraine with aura, epilepsy, traumatic brain injuries, transient global amnesia, stroke, and spinal cord diseases. Conclusion: Despite decades of research and hundreds of reports on the mechanism of spreading depression propagation, the exact mechanism of propagation still need to be elucidated. The present study reviews a group of these observations, in order to give some new insights into the complex mechanism of the propagation of spreading depression.

* Corresponding Author: Mahmoud Lotfinia

E-mail: mdla617@yahoo.com 


\title{
فرايند انتشار يديده مهار منتشر شونده: مرورى بر فرضيات مختلف
}

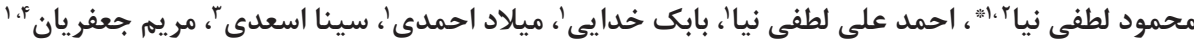 \\ مركز تحقيقات علوم اعصاب شفا، بيمارستان خاتم الانبياء، تهران، ايران.

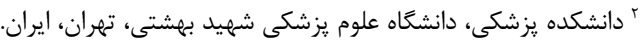

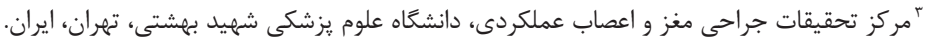

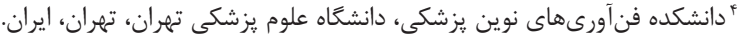

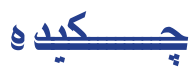

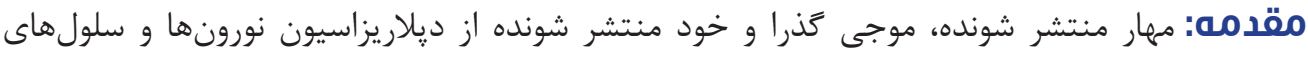

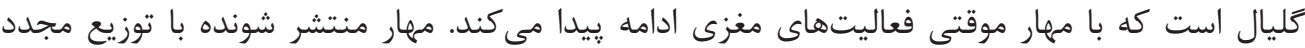

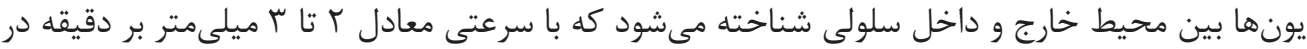

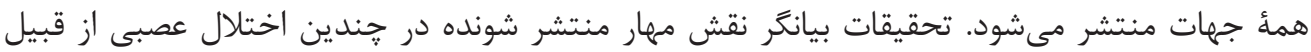

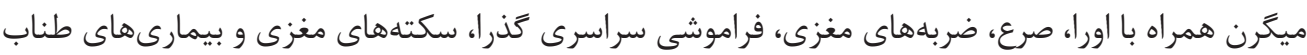

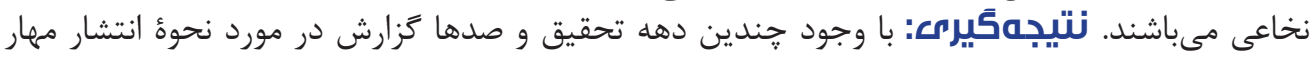

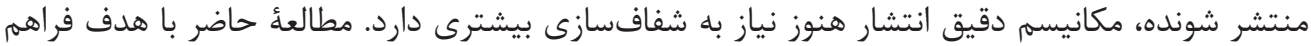

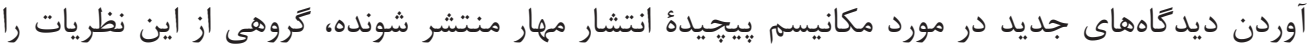
مرور مى كند.

ا ا مهار منتشر شونده قشرى

ب. ب. آستروسيتها r. اتصالات روزنه دار
" نويسنده مسئول: محمود لطفى نيا آدرس الكترونيكى: mdla617@yahoo.com 


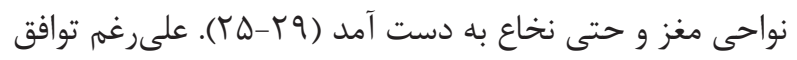

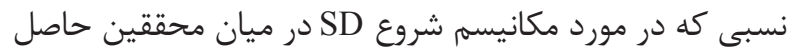

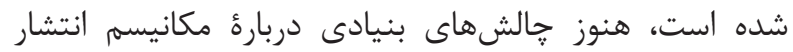

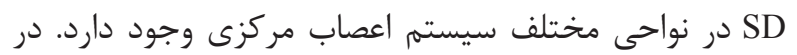

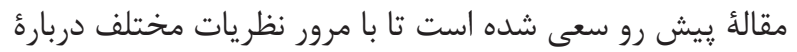

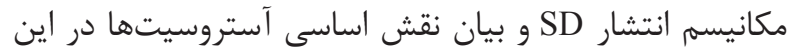

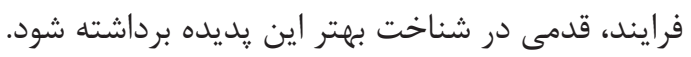

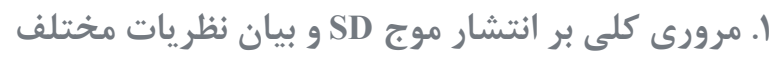

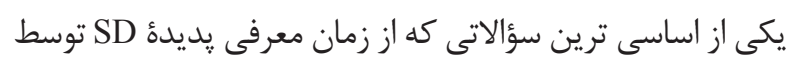

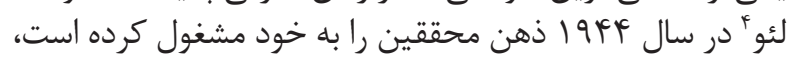

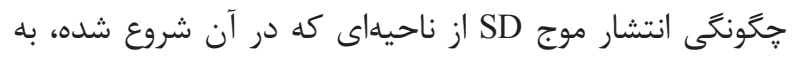

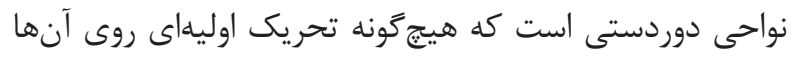

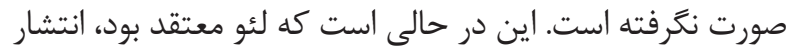

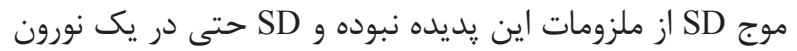

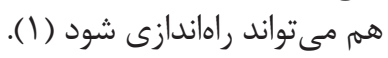

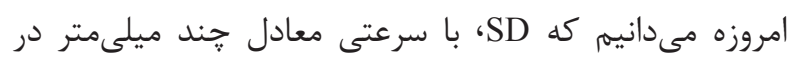

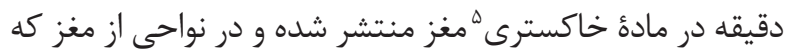

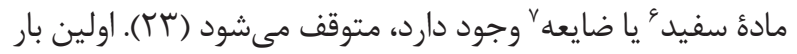

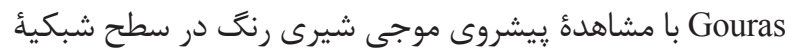

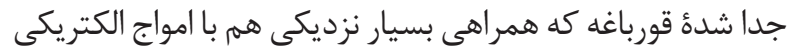

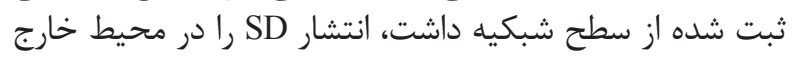

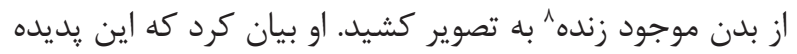

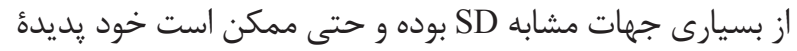

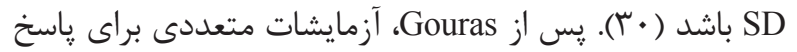

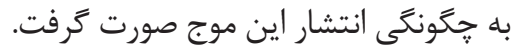

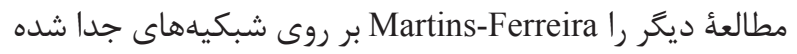

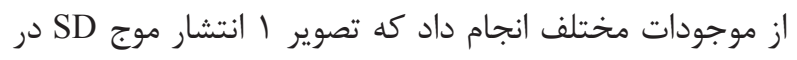

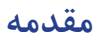

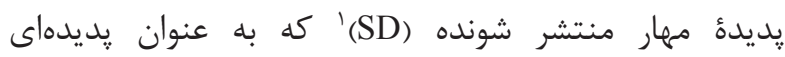

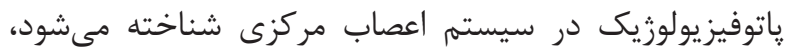

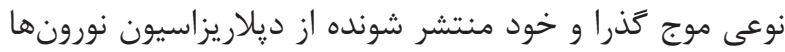

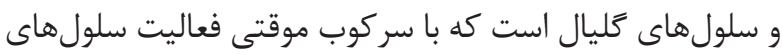

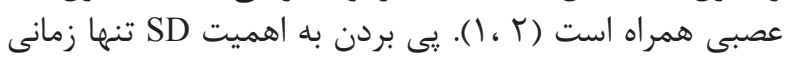

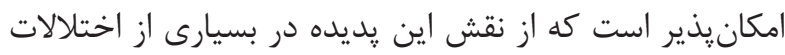

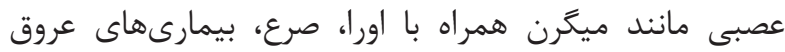

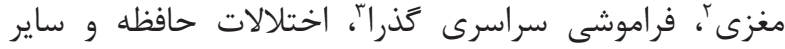

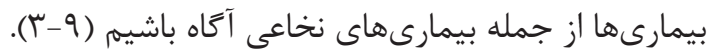

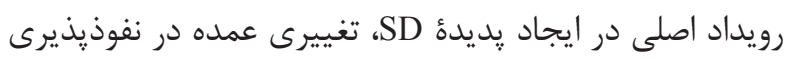

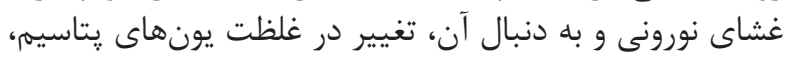

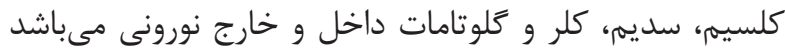

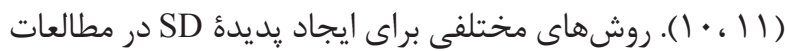

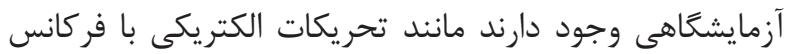

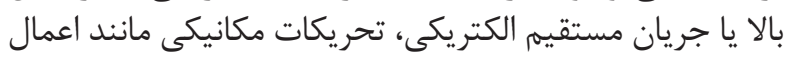

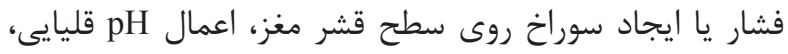

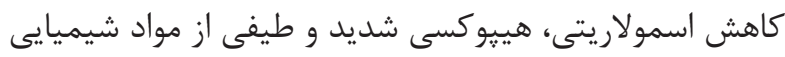

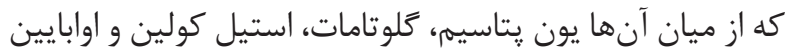

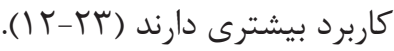

SD

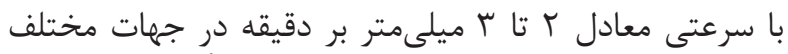

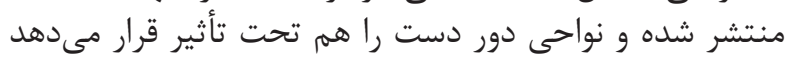

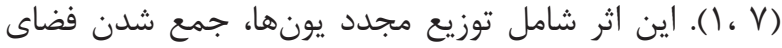

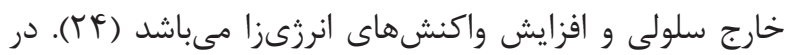

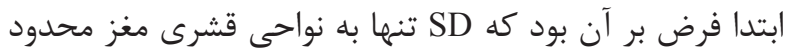

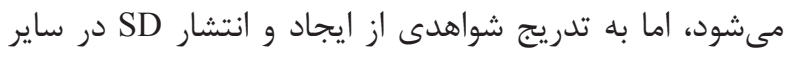
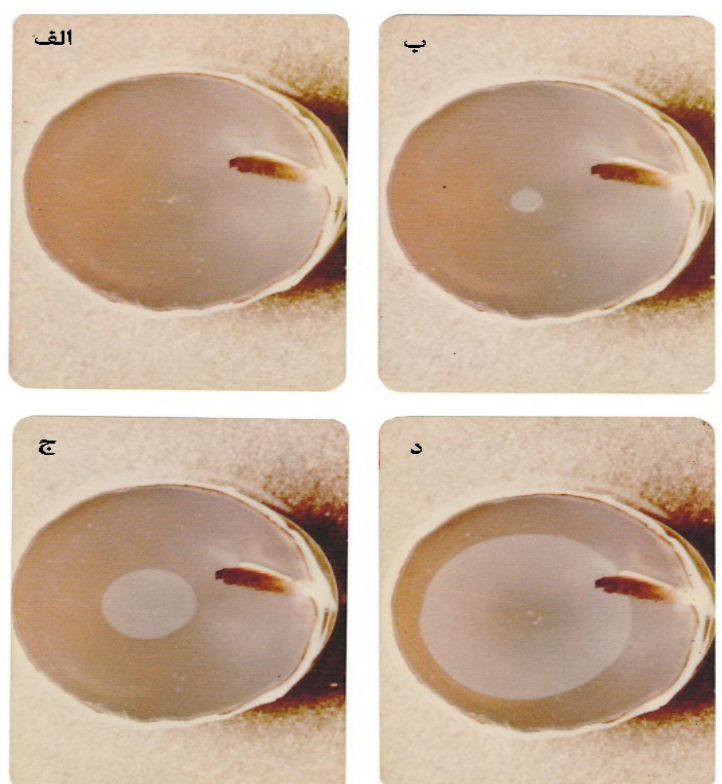

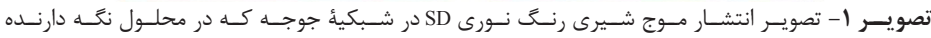

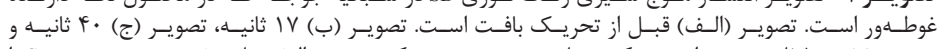

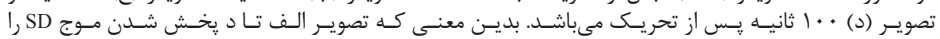

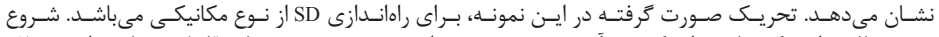

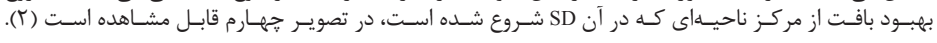

${ }^{1}$ Spreading depression

${ }^{2}$ Cerebrovascular diseases

${ }^{3}$ Transient global amnesia

\footnotetext{
${ }^{5}$ Gray matter

${ }^{6}$ White matter

${ }^{7}$ Scar

${ }^{8}$ In vitro
} 


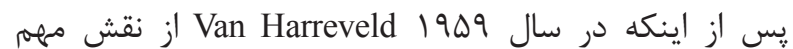

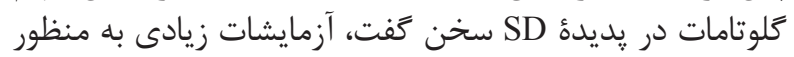

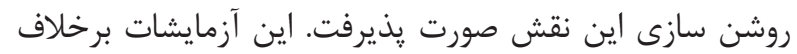

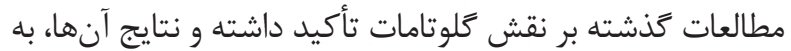

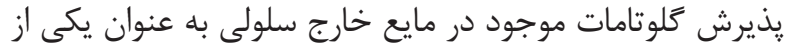

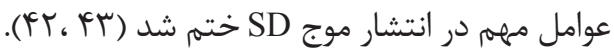

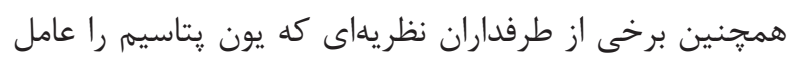

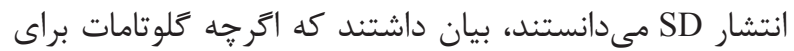

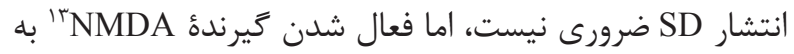

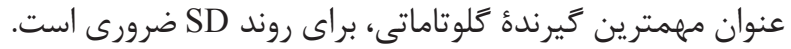

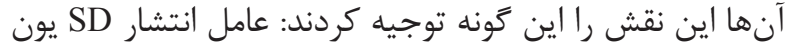

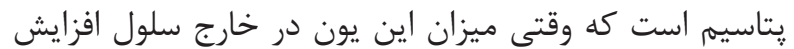

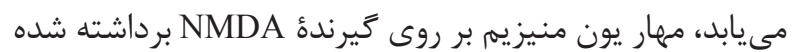

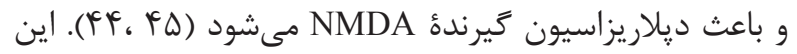

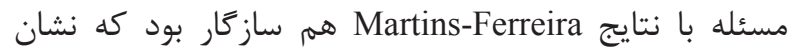

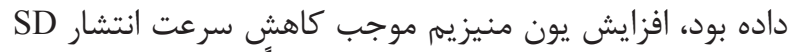

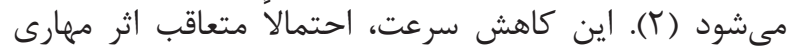

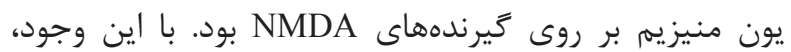

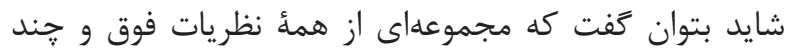

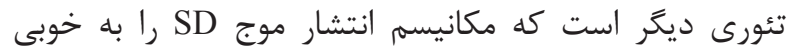

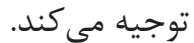

\section{r-1 - نقش اتصالات روزنه دار در انتشار SD}

يايه و اساس نسبت دهى انتشار SD به اتصالات روزنه دار راشايد

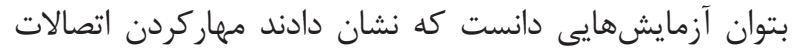

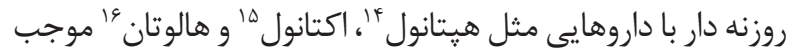

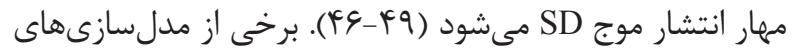

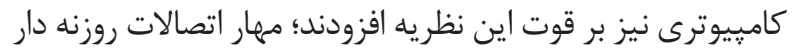

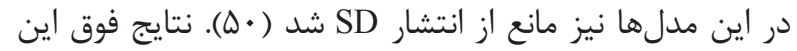

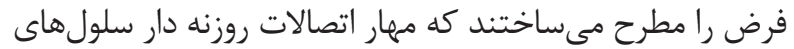

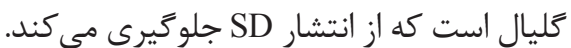

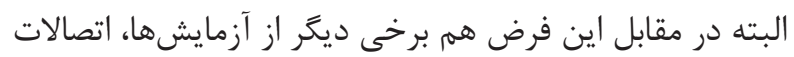

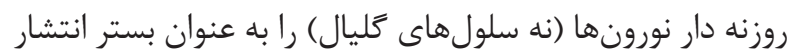

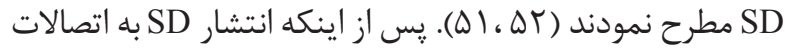

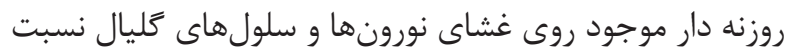

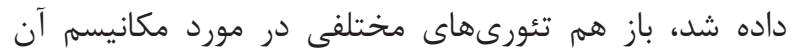

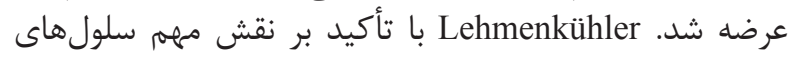

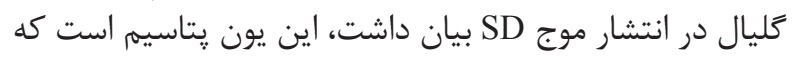

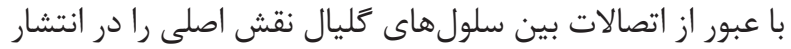

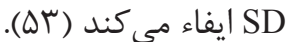

r. نقش آستروسيتها در جمع آورى و انتقال يون كلسيم، نظريةٌ موج كلسيمى آستروسيتى

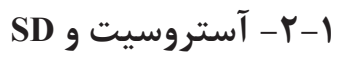

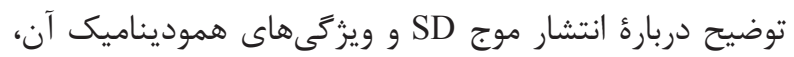

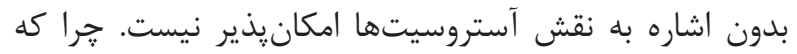

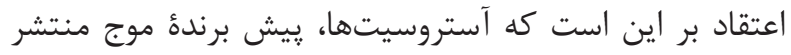

\footnotetext{
${ }^{9}$ Interstitial space

${ }^{10}$ Interstitial fluid

${ }^{11}$ Intercellular junctions

${ }^{12}$ Gap junctions
}

شبكيئ جوجه را نشان مىدهد (T). وى طيفى از يارامترهاى

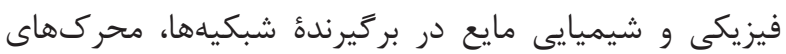

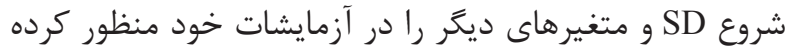

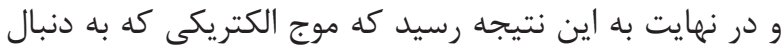

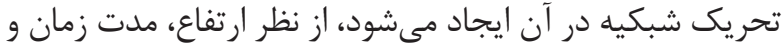

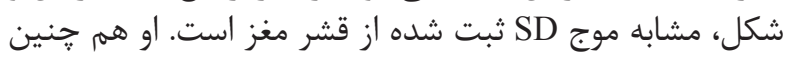

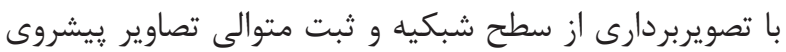

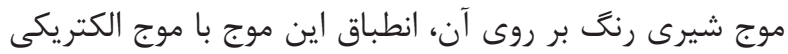

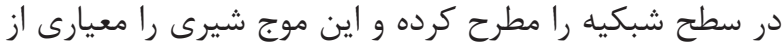

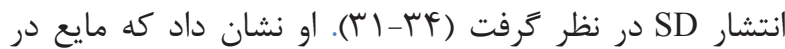

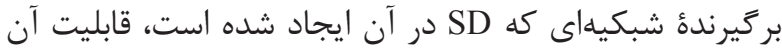

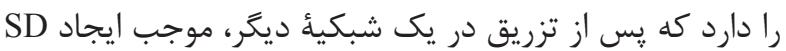

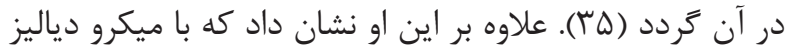

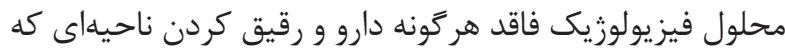
SD در آن جريان دارد، مىتوان از انتشار موج SD

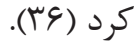

در مورد كليات جكَونكى انتشار ديلاريزاسيون ايجاد شده در يك ني

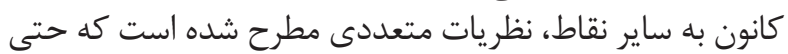

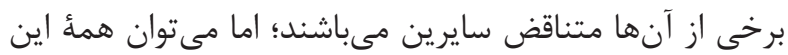

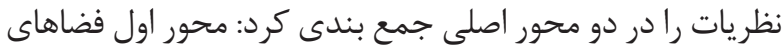

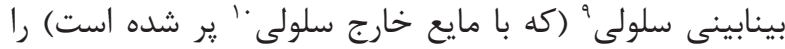

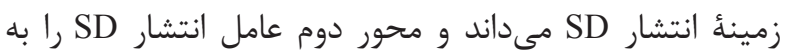

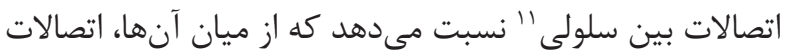
روزنه دارب" نقش حائز اهميتى دارند.

\section{|-1- مايع خارج سلولى و انتشار SD}

طرفداران نظريئ اول كه مايع خارج سلولى را بستر اصلى انتشار

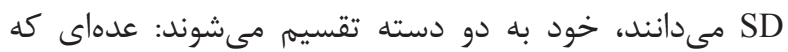

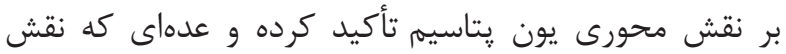

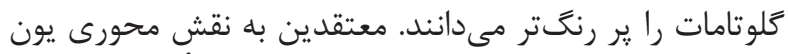

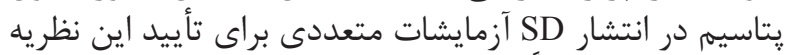

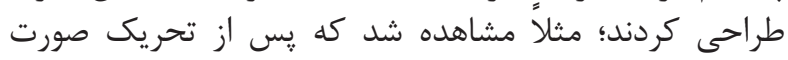

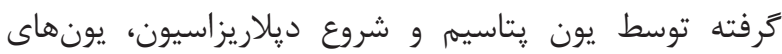

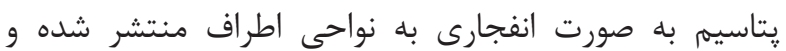

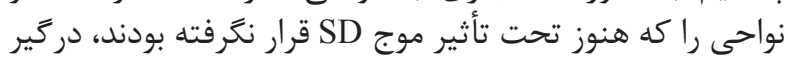

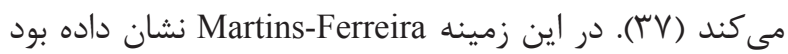

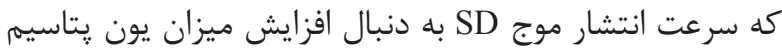

رو به افزايش مىنهيد (r) انتشار موج).

همجنين برخى ديكر هم نشان دادند كه ارتباطى بين آزاد شدن

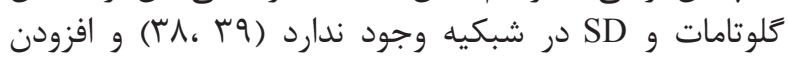

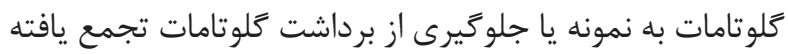

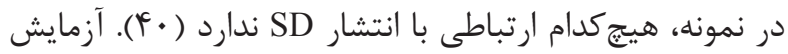

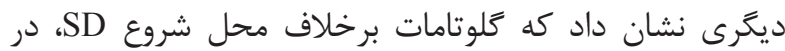

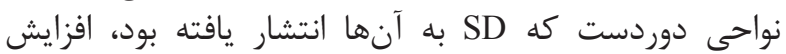

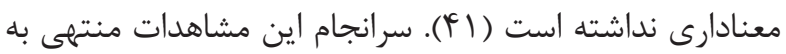

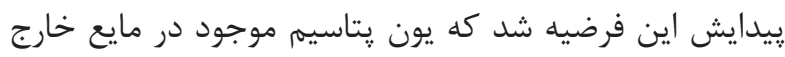

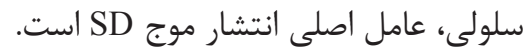

\footnotetext{
${ }^{13} \mathrm{~N}$-methyl D-aspartate

${ }^{14}$ Heptanol

${ }^{15}$ Octanol

${ }^{16}$ Halothane
} 
مىدهد. Dreier ناتوانى :مبٍ كلسيمى در خارج كردن كلسيم

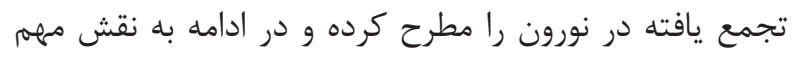

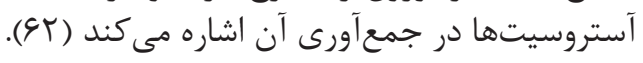
اين شايد دليلى باشد كه Cornell-Bell وجود يك سيستم

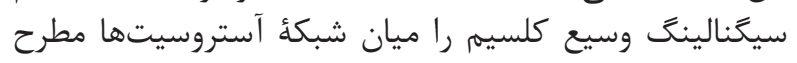

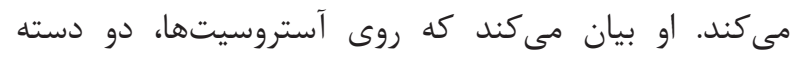

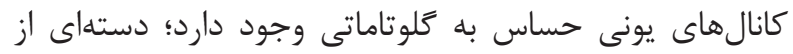

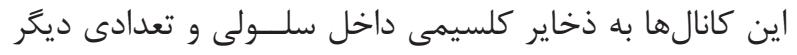

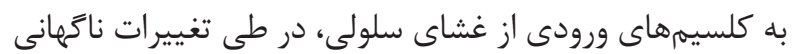

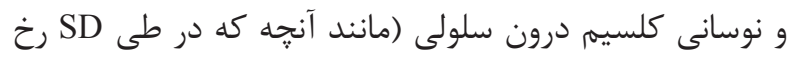

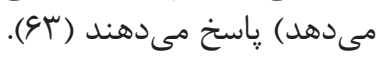

جند سال يس از ارائه نظريئ Cornell-Bell، خزارشاتى از

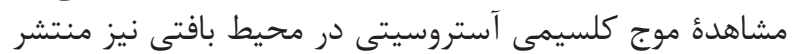

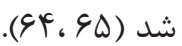

شونده SD مىباشند (r). آستروسيتها كه عموماً به عنوان

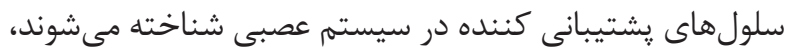

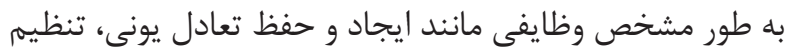

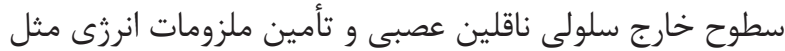

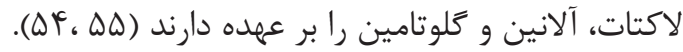
در طى دهdهاى اخير نيز، اختصاصاً بر قابليت ارتباط

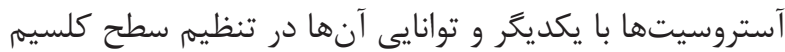

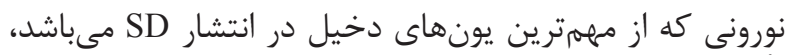

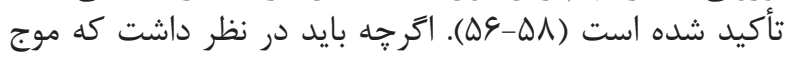

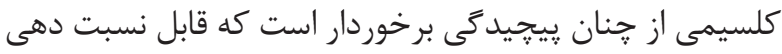

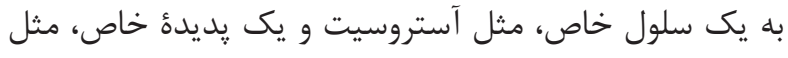
SD

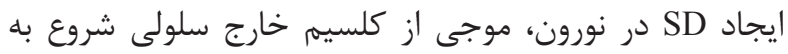

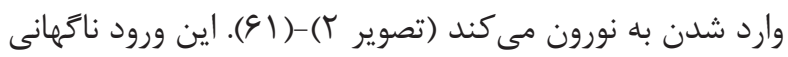

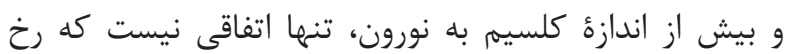
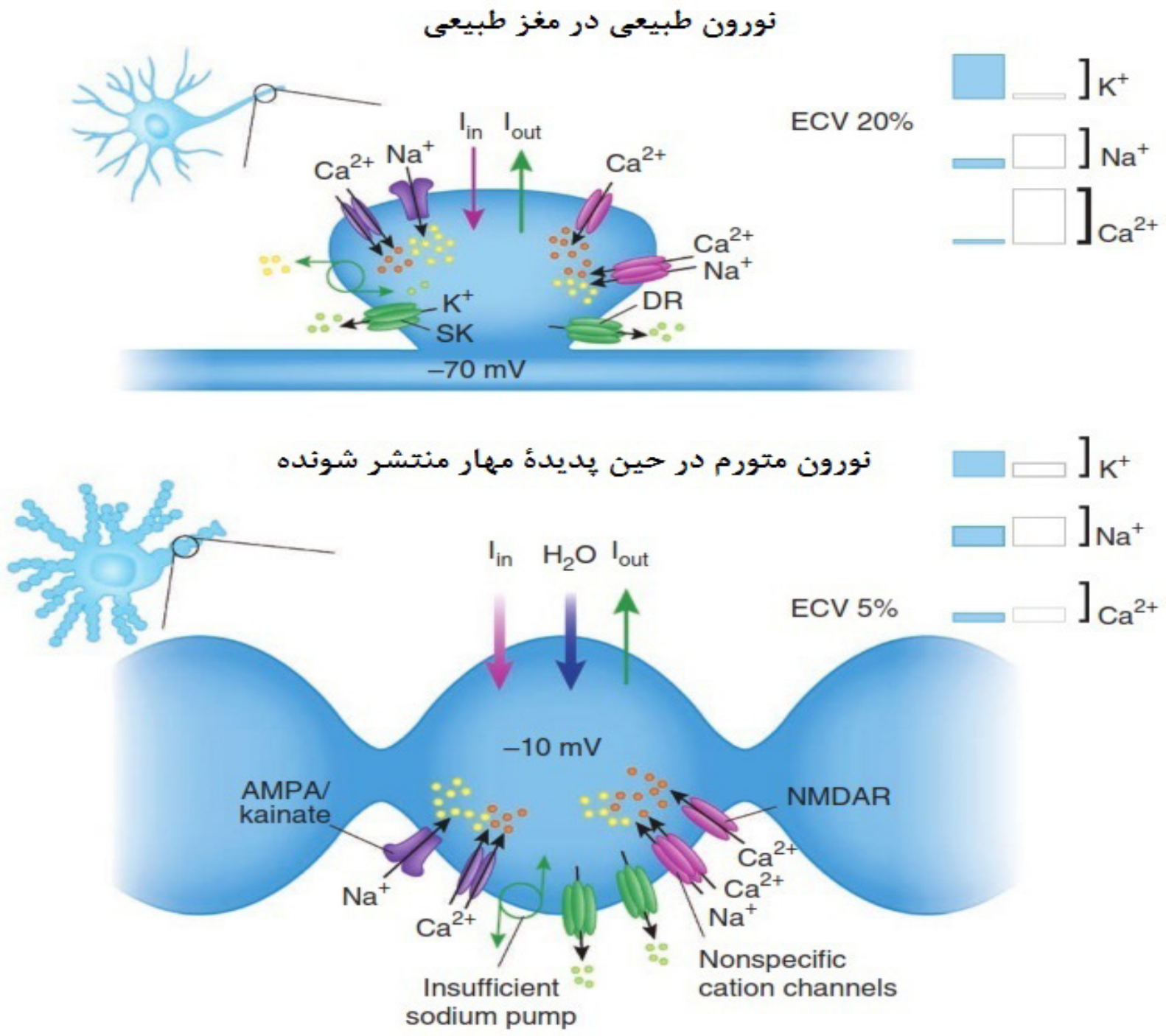

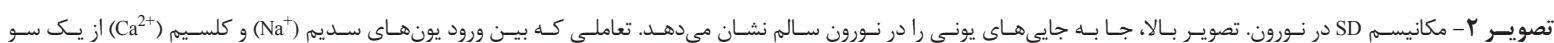

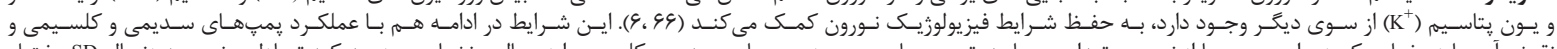

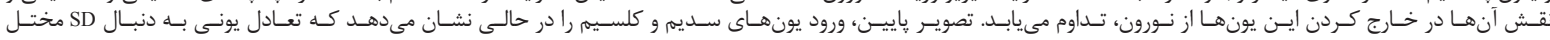

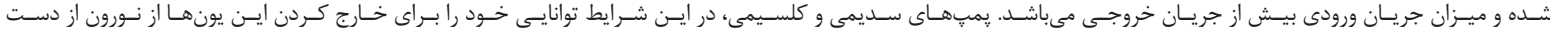

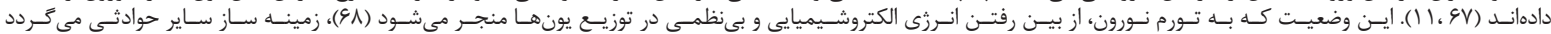

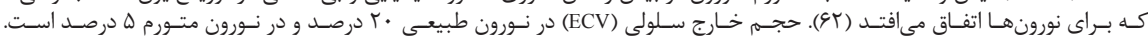




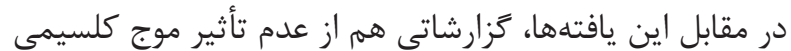

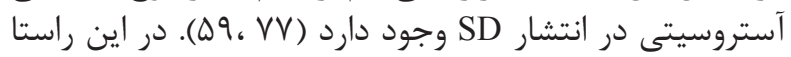
Basarsky

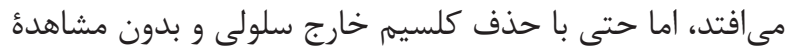

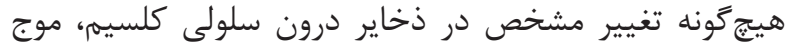

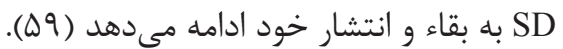

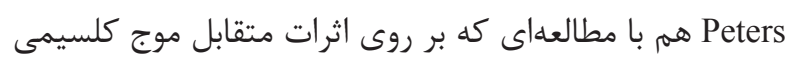

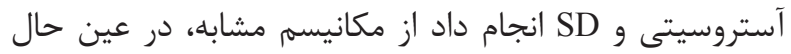

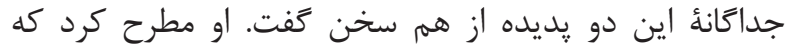

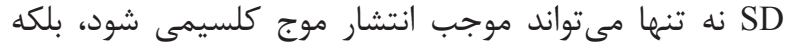

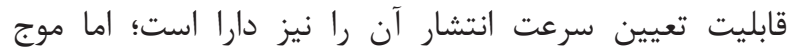

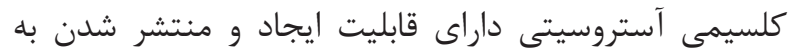

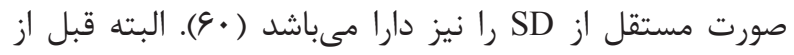
Peters

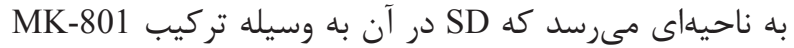

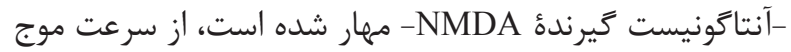

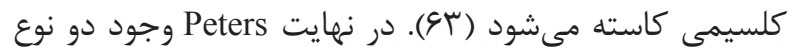

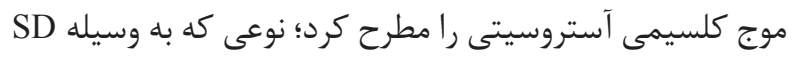

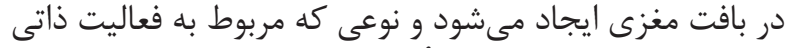

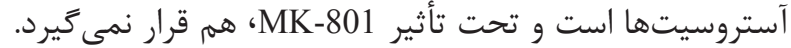

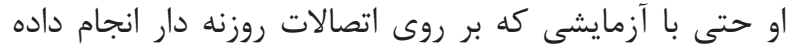

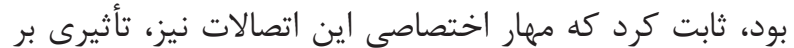

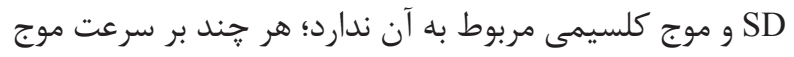

كلسيمى ناشى از فعاليت ذاتى آستروسيت ها نداردئ مؤثر بود (•؟).

دستهاى از مطالعات، از توقف انتشار SD به دنبال مهار اتصالات

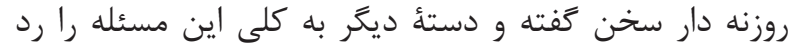

آستروسيت را در قبال عملكردش در برابر كلسيم كه نه فقط

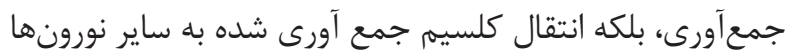

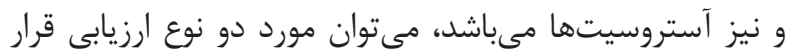

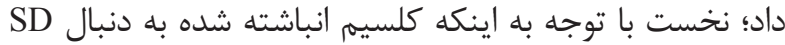

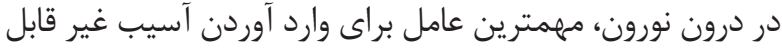

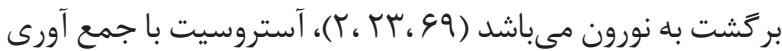

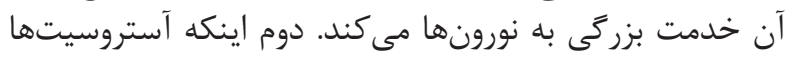

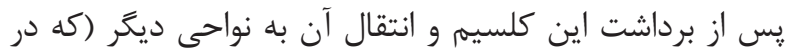

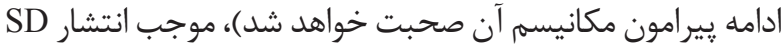

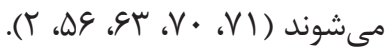

\section{r-r-r- موج كلسيمى آستروسيتى و SD}

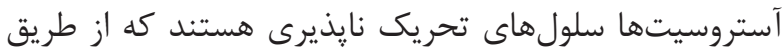

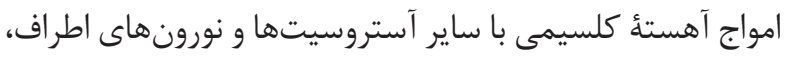

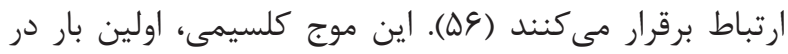

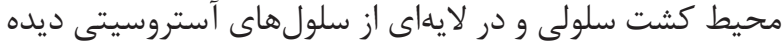

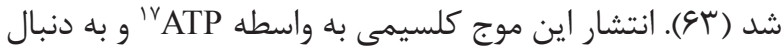

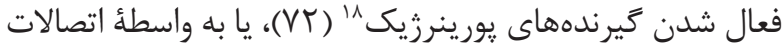

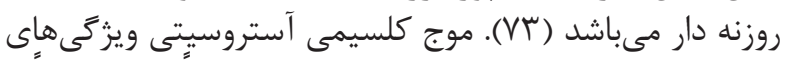

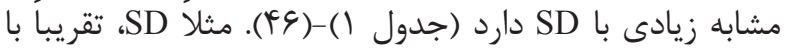

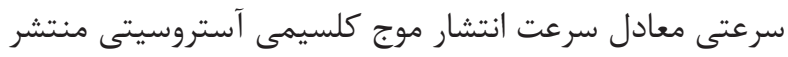

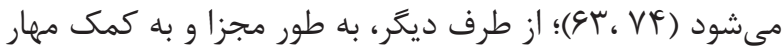

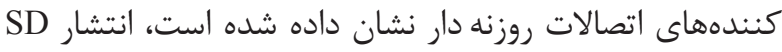

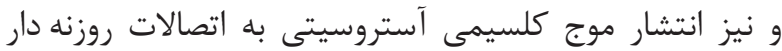

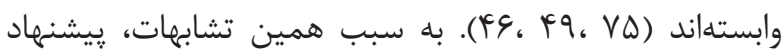

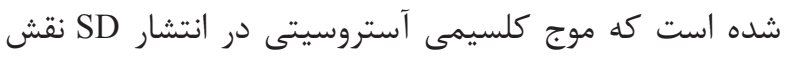
مؤثرى دارد (DG،VG).

جدول ا-مقايسٔ بين SD و موج آستروسيتى كلسيمى (r)

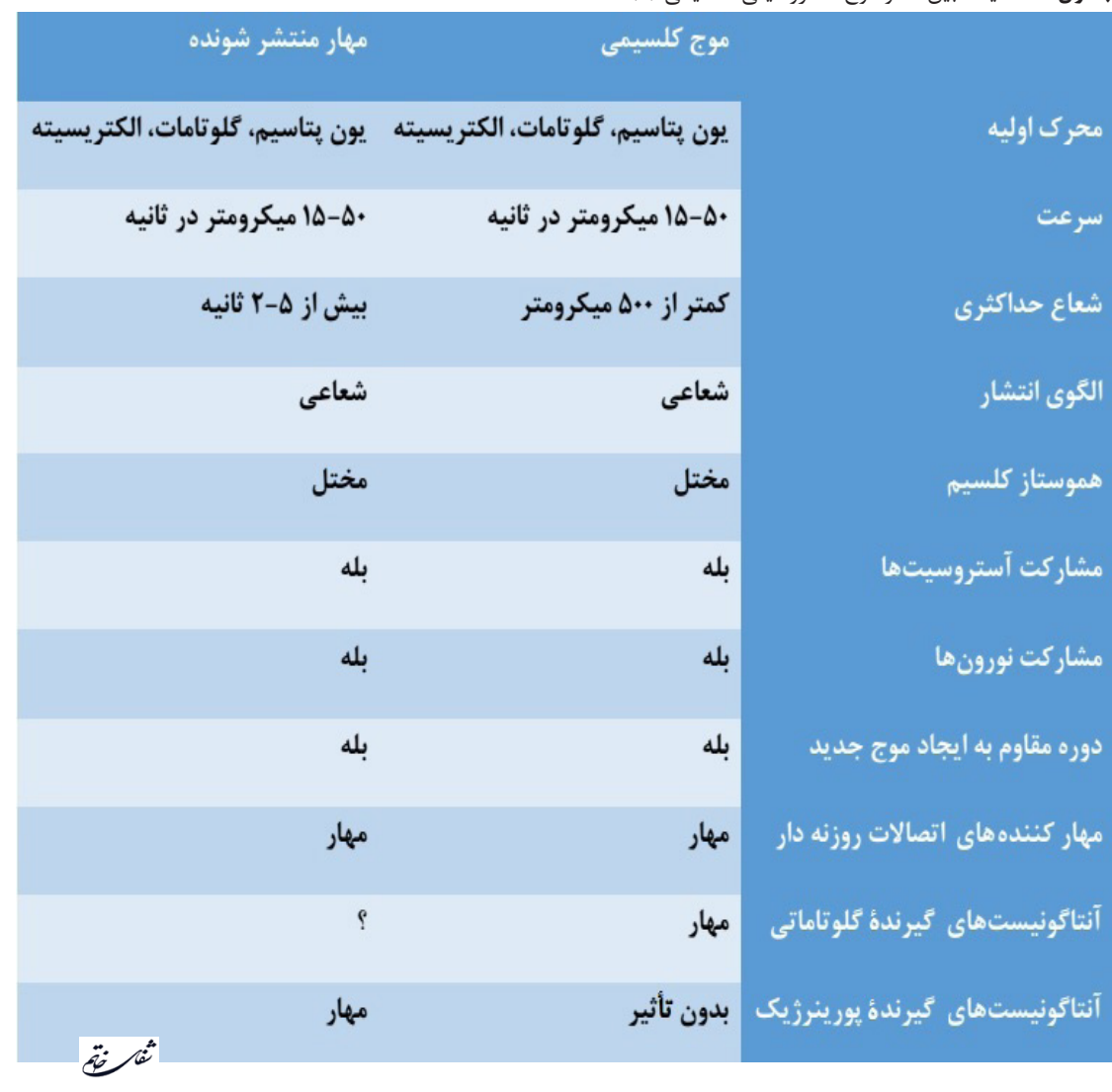

\footnotetext{
${ }^{17}$ Adenosine triphosphate
}

${ }^{18}$ Purinergic receptors 
نورون، در انتقال موج كلسيمى از سلول گليال به نورون اشاره

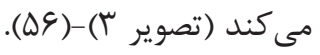

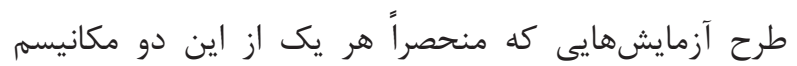

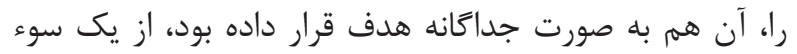

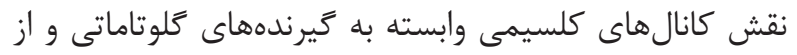

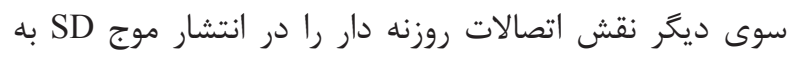

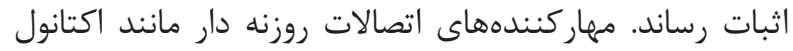

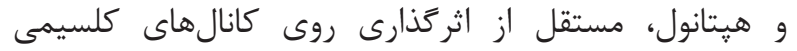

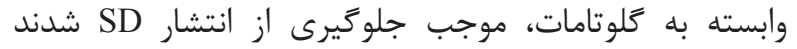

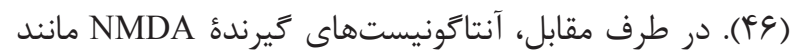

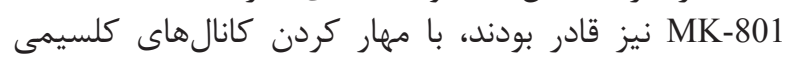

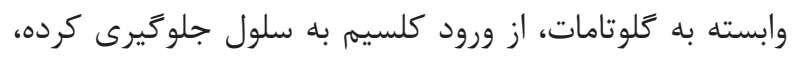

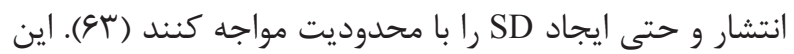

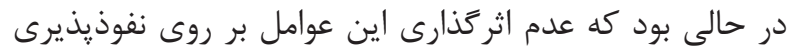

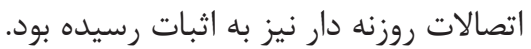

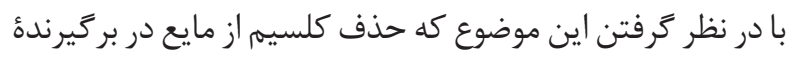

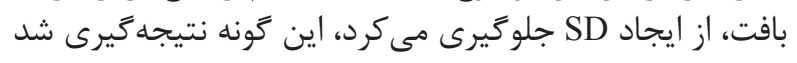

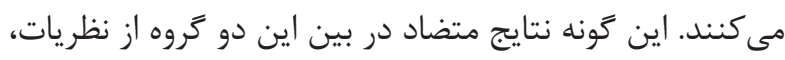

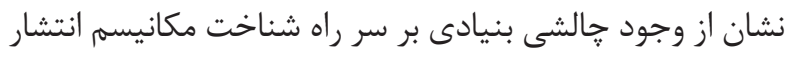

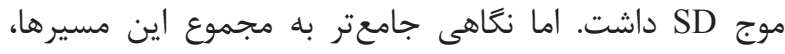

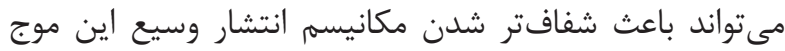
ديلاريزاسيون در سطح مغز شود.

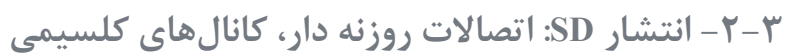
وابسته به تَلوتامات

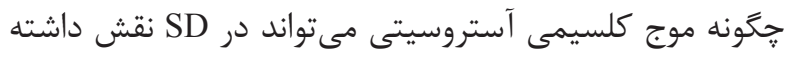

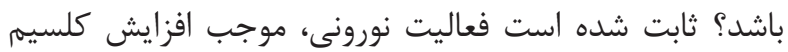

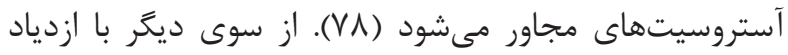

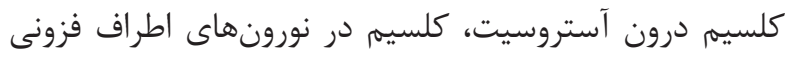

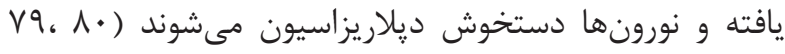

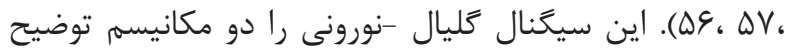

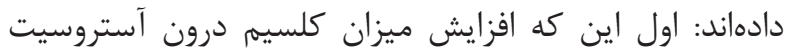

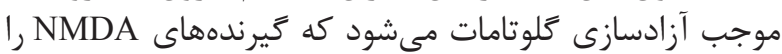

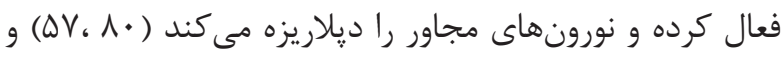

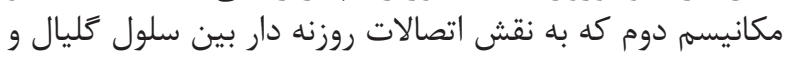

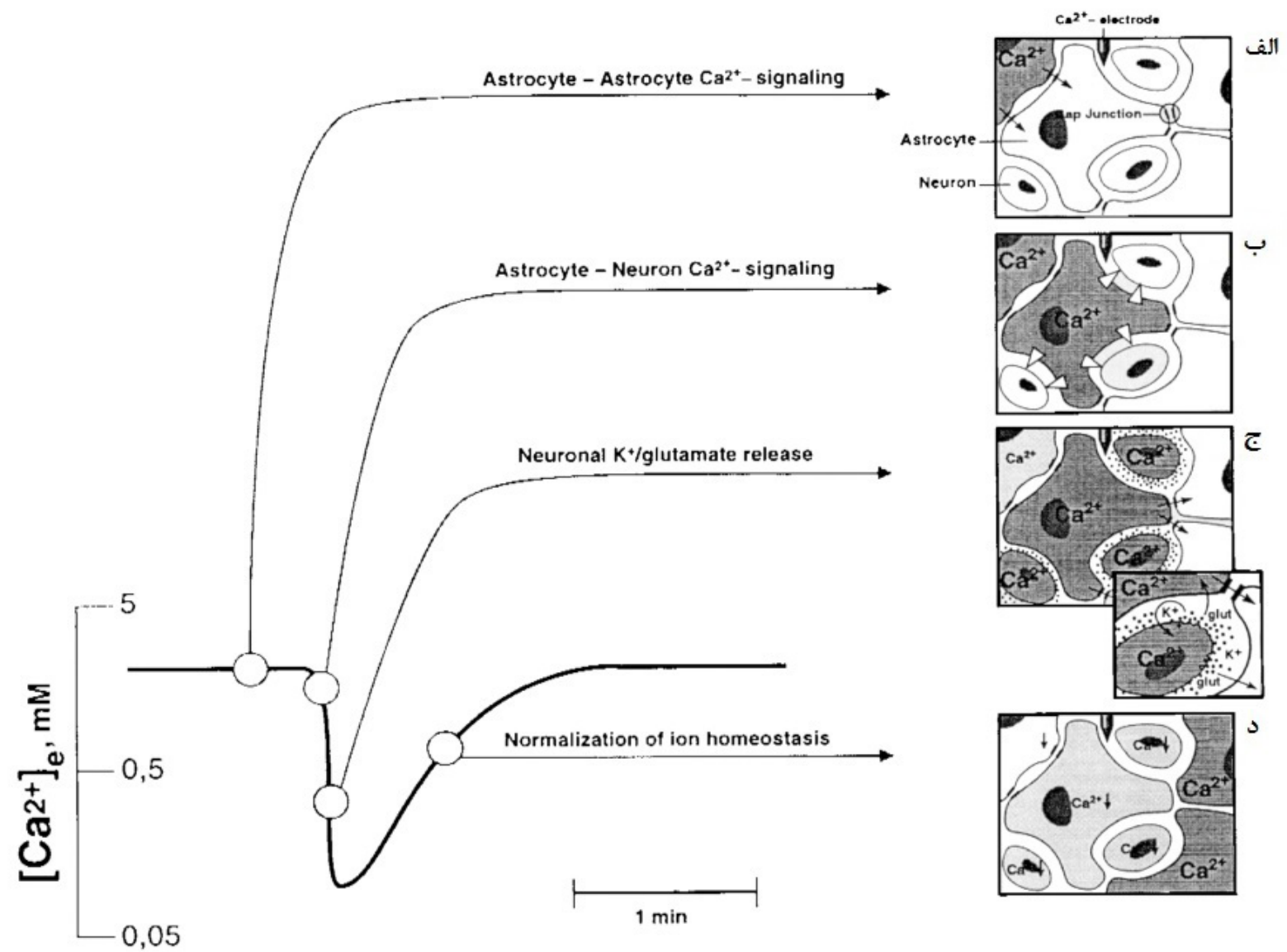

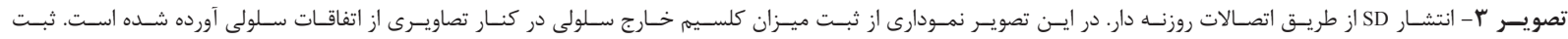

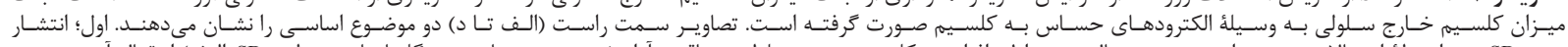

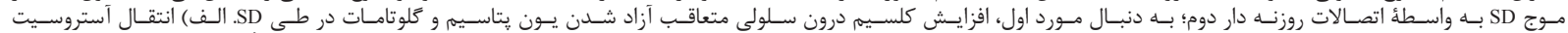

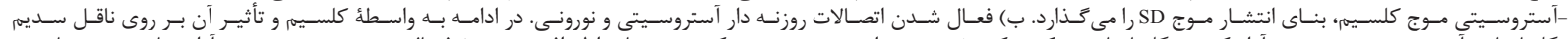

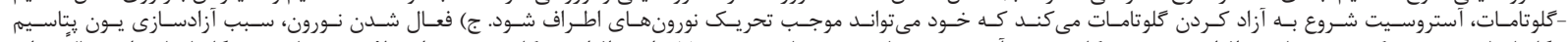

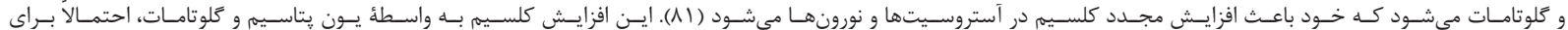

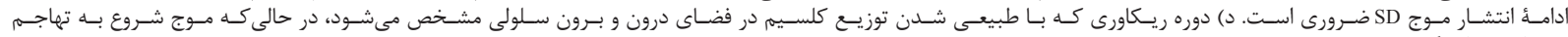


بسيارى از اختلالات عصبى مانند ميكرن همراه با اورا، صرع،

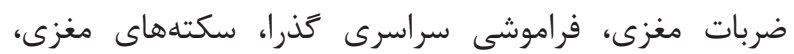
اختلالات حافظه و بيمارى هاى نخاعى نقش دارئ دارد. شناخت بهتر اين يديده، علاوه بر اينكه در روشن كردئ

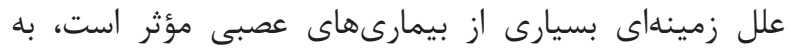

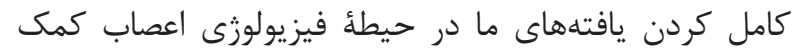

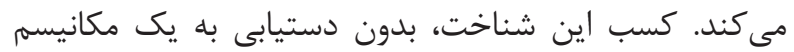

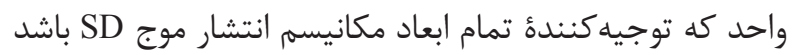

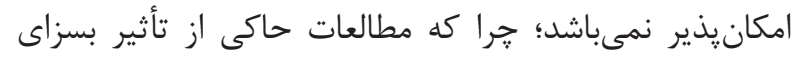

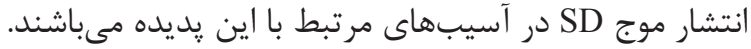
در حيطِهُ انتشار موج SD و در در آن دسته از مطالعاتى كه

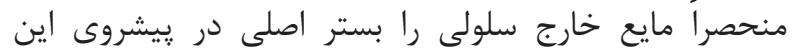

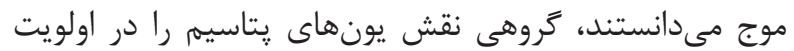

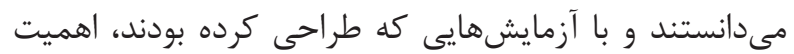

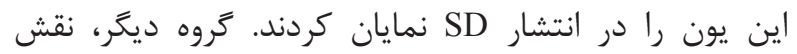

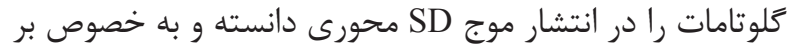

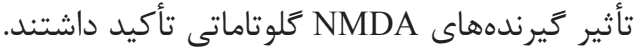

يس از مطرح شدن نقش احتمالى اتصالات روزنه دار در انتشار موج

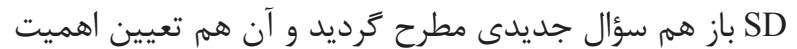

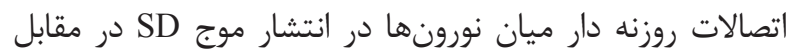

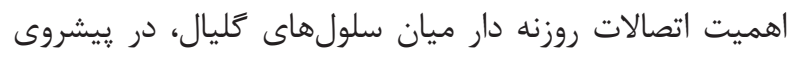

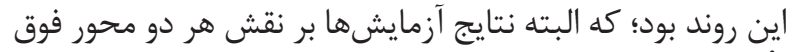

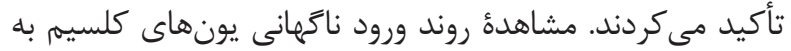

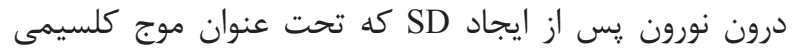

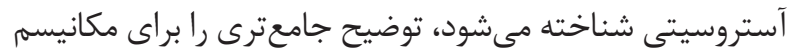

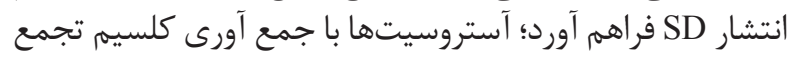

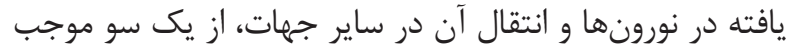

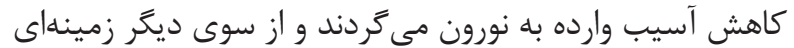

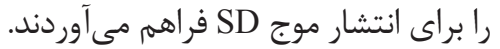

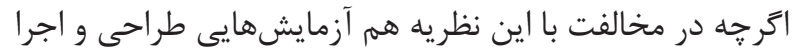

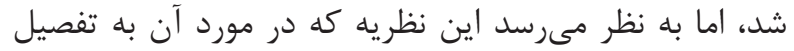

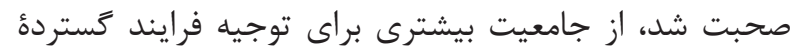

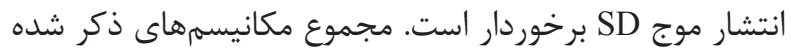

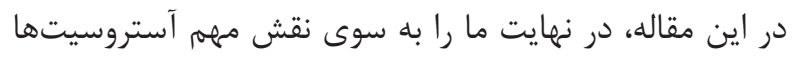

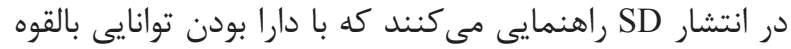

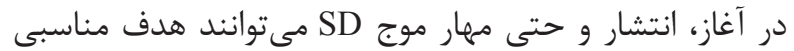

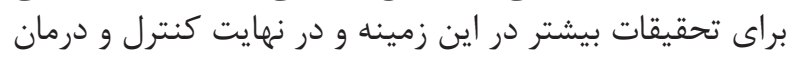

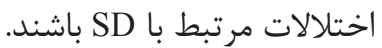

1. Leão AAP. Spreading depression of activity in the cerebral cortex. J Neurophysiol. 1944; 7(6): 359-90.

2. Martins-Ferreira $H$, Nedergaard $M$, Nicholson $C$. Perspectives on spreading depression. Brain Res Rev. 2000; 32(1): 215-34.

3. Busch E, Gyngell ML, Eis M, Hoehn-Berlage M,
كه فعال شدن كيرندههاى NMDA و به دنبال آن ورود كلسيم

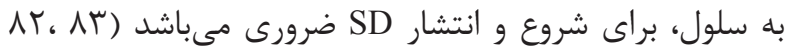

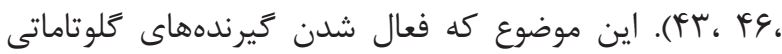

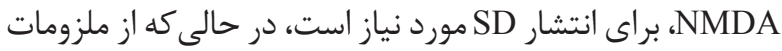

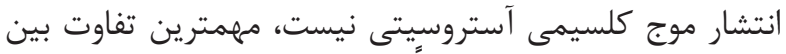

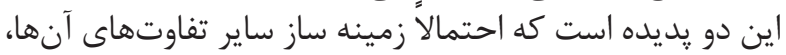

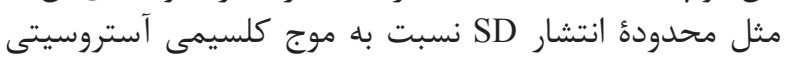

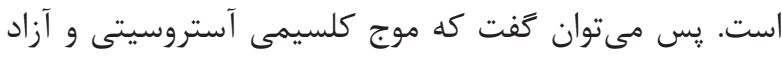

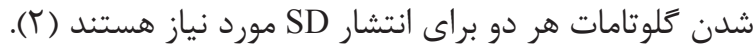

\section{SD نقش كانكسين Tr-Y}

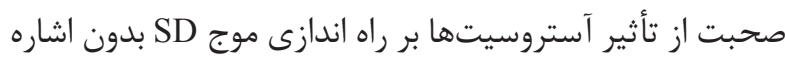

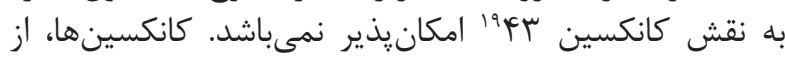

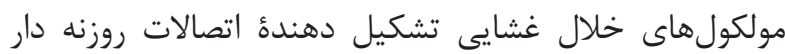

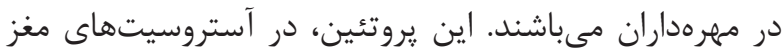

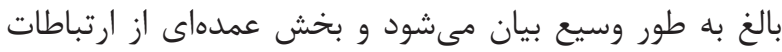

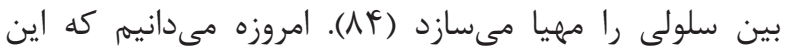

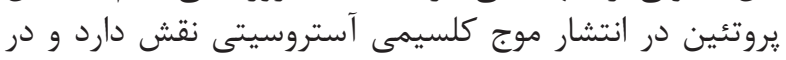

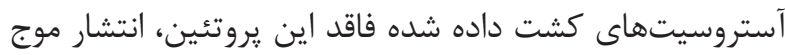

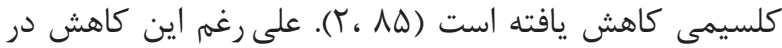

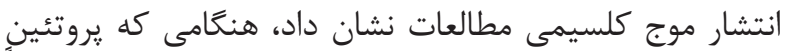

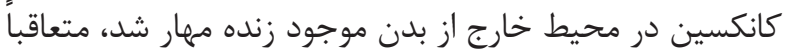

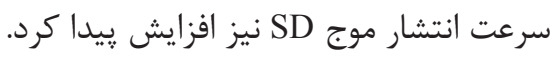

همانطور كه بيان شد، در طى روند SD مقادير قابل توجهى

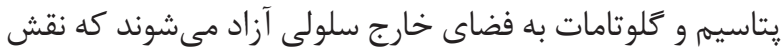

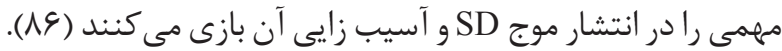

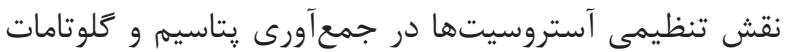

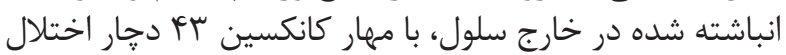

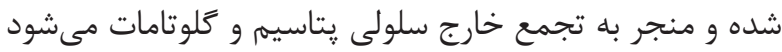
(AV، MN)

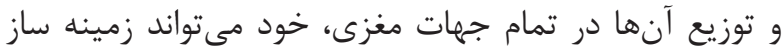

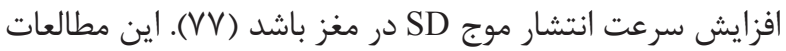

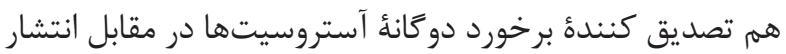

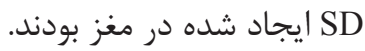

$$
\text { نتيجه كيرى }
$$

موجى كذرا و خود منتشر شونده از ديلاريزاسيون نورونها

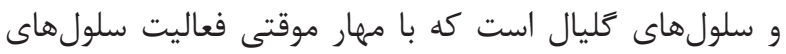

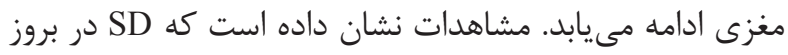

\section{منابع}

Hossmann K-A. Potassium-induced cortical spreading depressions during focal cerebral ischemia in rats: contribution to lesion growth assessed by diffusionweighted NMR and biochemical imaging. J Cereb Blood Flow Metab. 1996; 16(6): 1090-9.

4. Takano K, Latour LL, Formato JE, Carano RA, Helmer KG, Hasegawa Y, et al. The role of spreading 
depression in focal ischemia evaluated bv dffusion mapping. Ann Neurol. 2004; 39(3): 308-18.

5. Obeidat AS, Andrew RD. Spreading depression determines acute cellular damage in the hippocampal slice during oxygen/glucose deprivation. Eur J Neurosci. 2001; 10(11): 3451-61.

6. Lauritzen M. Pathophysiology of the migraine aura The spreading depression theory. Brain. 1994; 117(1): 199-210.

7. Gorji A. Spreading depression: a review of the clinical relevance. Brain Res Rev. 2001; 38(1-2): 33-60.

8. Lotfinia M. $\mathrm{Ca}^{2+}$ channel-blockers counter with the damaging effect of repetitive spreading depression on memory. J Neurol Sci. 2013; 333(1): e52.

9. Lotfinia M, Lotfinia A, Khodaie B, Ahmadi M. Spreading depression enhances the rate of neurogenesis in the rat hippocampus and dentate gyrus. Eur J Neurol; 2014; 21: 264.

10. Van Harreveld A. Two mechanisms for spreading depression in the chicken retina. J Neurobiol. 2004; 9(6): 419-31.

11. Kraio R, Nicholson C. Extracellular ionic variations during spreading depression. Neuroscience. 1978; 3(11): 1045-59.

12. Balestrino M. Studies on anoxic depolarization. Rigor BM, Schurr A. Brain Slices in Basic and Clinical Research. CRC, Boca Raton, FL. 1995; p.273-93.

13. Balestrino M, Young J, Aitken P. Block of $\left(\mathrm{Na}^{+}, \mathrm{K}^{+}\right)$ ATPase with ouabain induces spreading depression-like depolarization in hippocampal slices. Brain Res. 1999; 838(1): 37-44.

14. Bures J, Buresova O, Krivanek J. The mechanism and applications of Leao's spreading depression of EEG activity. New York: Academic. 1974.

15. Chebabo S, Hester M, Aitken P, Somjen G. Hypotonic exposure enhances synaptic transmission and triggers spreading depression in rat hippocampal tissue slices. Brain Res. 1995; 695(2): 203-16.

16. El-Bachá R, Lima-Filho J, Guedes R. Dietary antioxidant deficiency facilitates cortical spreading depression induced by photoactivated riboflavin. Nutr Neurosci. 1998;1(3):205-12.

17. Guedes RCA, Carmo RJ. Influence of ionic disturbances produced by gastric washing on cortical spreading depression. Exp Brain Res. 1980; 39(3): 341-9.

18. Lauritzen M, Rice ME, Okada Y, Nicholson C. Quisqualate, kainate and NMDA can initiate spreading depression in the turtle cerebellum. Brain Res. 1988; 475(2): 317-27.

19. Marshall WH. Spreading cortical depression of Leao. Physiol Rev. 1959; 39(2): 239-79.

20. Ochs S. The nature of spreading depression in neural networks. Int Rev Neurobiol. 1962; 4: 1-69.

21. Reid KH, Marrannes R, Wauquier A. Spreading depression and central nervous system pharmacology. J Pharmacol Methods. 1988; 19(1): 1-21.

22. Roitbak A, Bobrov A. Spreading depression resulting from cortical punctures. Acta Neurobiol Exp (Wars). 1975; 35: 761-8.

23. Somjen GG. Mechanisms of spreading depression and hypoxic spreading depression-like depolarization. Physiol Rev. 2001; 81(3): 1065-96.

24. Nicholson C, Kraig R. The behavior of extracellular ions during spreading depression. The application of ion-selective microelectrodes. 1981: p. 217-38.

25. Streit D, Ferreira C, Martins-Ferreira H. Spreading depression in isolated spinal cord. J Neurophysiol. 1995; 74: 888-90.

26. Gorji A, Zahn P, Pogatzki E, Speckmann E-J. Spinal and cortical spreading depression enhance spinal cord activity. Neurobiol Dis. 2004; 15(1): 70-9.

27. Seghatoleslam M, Ghadiri MK, Ghaffarian N, Speckmann E-J, Gorji A. Cortical spreading depression modulates the caudate nucleus activity. Neuroscience. 2014; 267: 83-90.

28. Martens-Mantai T, Speckmann EJ, Gorji A. Propagation of cortical spreading depression into the hippocampus: The role of the entorhinal cortex. Synapse. 2014; doi: 10.1002/syn. 21769.

29. Fifková E. Thalamic spreading depression in the rat. Electroencephalogr Clin Neurophysiol. 1966; 20(1): 6876.

30. Gouras P. Spreading depression of activity in amphibian retina. Am J Physiol. 1958; 195(1): 28-32.

31. Martins-Ferreira H. Spreading depression in the chicken retina. The brain and behavior of the fowl Japan Scientific Society Press, Tokyo. 1983: 317-33. 
32. Martins-Ferreira H. Propagation of spreading depressioninisolated retina.Migraine:BasicMechanisms and Treatments, Urban and Schwartzenberg, Munich. 1993: 533-46.

33. Martins-Ferreira H, de Castro GO. Light-scattering changes accompanying spreading depression in isolated retina. J Neurophysiol. 1966; 29(4): 715-26.

34. Martins-Ferreira H, Castro GDO, Struchiner C, Rodrigues P. Circling spreading depression in isolated chick retina. J Neurophysiol. 1974 ; 37(4): 773-84.

35. Martins-Ferreira H, Castro GDO, Stuchiner C, Rodrigues P. Liberation of chemical factors during spreading depression in isolated retina. J Neurophysiol. 1974; 37(4): 785-91.

36. Obrenovitch TP, Zilkha E. Intracerebral Microdialysis Markedly Inhibits the Propagation of Cortical Spreading Depression. Clinical Aspects of Microdialysis. Springer. 1996; p. 21-3.

37. Grafstein B. Mechanism of spreading cortical depression. J Neurophysiol. 1956; 19(2): 154-71.

38. Carmo R, Leao A. On the relation of glutamic acid and some allied compounds to cortical spreading depression. Brain Res. 1972; 39(2): 515-18.

39. Gardino PF, Do Carmo R. Glutamate and spreading depression in chick retina. An Acad Bras Cienc. 1983; 55(3): 297-307.

40. Somjen GG, Tombaugh GC. $\mathrm{pH}$ modulation of neuronal excitability and central nervous system functions. $\mathrm{pH}$ and Brain Function. 1998: 373-93.

41. Scheller D, Heister U, Kolb J, Tegtmeier F. On the role of excitatory amino acids during generation and propagation of spreading depression. Migraine: Basic Mechanisms and Treatment, eds Lehmenkühler A, Grotemeyer KH, Tegtmeier F (Urban \& Schwarzenberg, München, Germany). 1993: 355-66.

42. Van Harreveld A. Two mechanisms for spreading depression in the chicken retina. J Neurobiol. 1978; 9(6): 419-31.

43. Marrannes R, Willems R, De Prins E, Wauquier A. Evidence for a role of the N-methyl-d-aspartate (NMDA) receptor in cortical spreading depression in the rat. Brain Res. 1988; 457(2): 226-40.

44. Obrenovitch T, Zilkha E. Inhibition of cortical spreading depression by L-701,324, a novel antagonist at the glycine site of the N-methyl-D-aspartate receptor complex. Br J Pharmacol. 1996; 117(5): 931-37.

45. Obrenovitch TP, Zilkha E, Urenjak J. Evidence against high extracellular glutamate promoting the elicitation of spreading depression by potassium. J Cereb Blood Flow Metab. 1996; 16(5): 923-31.

46. Nedergaard M, Cooper AJ, Goldman SA. Gap junctions are required for the propagation of spreading depression. J Neurobiol. 1995; 28(4): 433-44.

47. Rawanduzy A, Hansen A, Hansen TW, Nedergaard M. Effective reduction of infarct volume by gap junction blockade in a rodent model of stroke. J Neurosurg. 1997; 87(6): 916-20.

48. Piper R, Lambert G. Inhalational anesthetics inhibit spreading depression: relevance to migraine. Cephalalgia. 1996; 16(2): 87-92.

49. Martins-Ferreira H, Ribeiro L. Biphasic effects of gap junctional uncoupling agents on the propagation of retinal spreading depression. Braz J Med Biol Res. 1995; 28(9): 991-4.

50. Shapiro BE. Osmotic forces and gap junctions in spreading depression: a computational model. J Comput Neurosci. 2001; 10(1): 99-120.

51. Largo C, Tombaugh GC, Aitken PG, Herreras O, Somjen GG. Heptanol but not fluoroacetate prevents the propagation of spreading depression in rat hippocampal slices. J Neurophysiol. 1997; 77(1): 9-16.

52. Herreras O, Largo C, Ibarz J, Somjen G, del Rio $\mathrm{RM}$. Role of neuronal synchronizing mechanisms in the propagation of spreading depression in the in vivo hippocampus. J Neurosci. 1994; 14(11): 7087-98.

53. Lehmenkuêhler A. Spreading depressionReaktionen an der Hirnrinde: Störungen des extrazellulären Mikromilieus. EEG-EMG Zeitschrift für Elektroenzephalographie, Elektromyographie und verwandte Gebiete. 1990; 21(1): 1-6.

54. Stewart RM, Rosenberg RN. Physiology of glia: glial-neuronal interactions. Int Rev Neurobiol. 1979; 21: $275-309$.

55. Simard M, Nedergaard M. The neurobiology of glia in the context of water and ion homeostasis. Neuroscience. 2004; 129(4): 877-96.

56. Nedergaard M. Direct signaling from astrocytes to neurons in cultures of mammalian brain cells. Science. 
1994; 263(5154): 1768-71.

57. Parpura V, Basarsky TA, Liu F, Jeftinija K, Jeftinija S, Haydon PG. Glutamate-mediated astrocyte-neuron signalling. Nature. 1994; 369: 744 - 7.

58. Jing J, Aitken PG, Somjen GG. Role of calcium channels in spreading depression in rat hippocampal slices. Brain Res. 1993; 604(1): 251-9.

59. Basarsky TA, Duffy SN, Andrew RD, MacVicar BA. Imaging spreading depression and associated intracellular calcium waves in brain slices. J Neurosci. 1998; 18(18): 7189-99.

60. Peters O, Schipke CG, Hashimoto Y, Kettenmann H. Different mechanisms promote astrocyte $\mathrm{Ca}^{2+}$ waves and spreading depression in the mouse neocortex. J Neurosci. 2003; 23(30): 9888-96.

61. Canals S, Makarova I, Lopez-Aguado L, Largo C, Ibarz JM, Herreras O. Longitudinal depolarization gradients along the somatodendritic axis of CA1 pyramidal cells: a novel feature of spreading depression. J Neurophysiol. 2005; 94(2): 943-51.

62. Dreier JP. The role of spreading depression, spreading depolarization and spreading ischemia in neurological disease. Nat Med. 2011; 17(4): 439-47.

63. Cornell-Bell AH, Finkbeiner SM, Cooper MS, Smith SJ. Glutamate induces calcium waves in cultured astrocytes: long-range glial signaling. Science. 1990; 247(4941): 470-3.

64. Schipke CG, Boucsein C, Ohlemeyer C, Kirchhoff F, Kettenmann H. Astrocyte $\mathrm{Ca}^{2+}$ waves trigger responses in microglial cells in brain slices. FASEB J. 2002; 16(2): 255-7.

65. Newman EA, Zahs KR. Calcium waves in retinal glial cells. Science. 1997; 275(5301): 844-7.

66. Windmüller O, Lindauer U, Foddis M, Einhäupl $\mathrm{KM}$, Dirnagl U, Heinemann U, et al. Ion changes in spreading ischaemia induce rat middle cerebral artery constriction in the absence of NO. Brain. 2005; 128(9): 2042-51.

67. Kager H, Wadman W, Somjen G. Conditions for the triggering of spreading depression studied with computer simulations. J Neurophysiol. 2002; 88(5): 2700-12.

68. Takano T, Tian G-F, Peng W, Lou N, Lovatt D, Hansen AJ, et al. Cortical spreading depression causes and coincides with tissue hypoxia. Nat Neurosci. 2007; 10(6): 754-62.
69. Dietz RM, Weiss JH, Shuttleworth CW. $\mathrm{Zn}^{2+}$ influx is critical for some forms of spreading depression in brain slices. J Neurosci. 2008; 28(32): 8014-24.

70. Finkbeiner SM. Glial calcium. Glia. 1993; 9(2): 83-104.

71. Glaum SR, Holzwarth JA, Miller RJ. Glutamate receptors activate $\mathrm{Ca}^{2+}$ mobilization and $\mathrm{Ca}^{2+}$ influx into astrocytes. Proc Natl Acad Sci USA. 1990; 87(9): 3454-8.

72. Guthrie PB, Knappenberger J, Segal M, Bennett MV, CharlesAC,KaterS.ATPreleased fromastrocytesmediates glial calcium waves. J Neurosci. 1999; 19(2): 520-8.

73. Giaume C, Venance L. Intercellular calcium signaling and gap junctional communication in astrocytes. Glia. 1998; 24(1): 50-64.

74. Hansen A. Effect of anoxia on ion distribution in the brain. Physiol Rev. 1985; 65(1): 101-48.

75. Finkbeiner S. Calcium waves in astrocytes-filling in the gaps. Neuron. 1992; 8(6): 1101-8.

76. Cornell-Bell A, Finkbeiner S. $\mathrm{Ca}^{2+}$ waves in astrocytes. Cell Calcium. 1991; 12(2): 185-204.

77. Theis M, Jauch R, Zhuo L, Speidel D, Wallraff A, Döring B, et al. Accelerated hippocampal spreading depression and enhanced locomotory activity in mice with astrocyte-directed inactivation of connexin43. J Neurosci. 2003; 23(3): 766-76.

78. Dani JW, Chernjavsky A, Smith SJ. Neuronal activity triggers calcium waves in hippocampal astrocyte networks. Neuron. 1992; 8(3): 429-40.

79. Charles A. Glia-neuron intercellular calcium signaling. Dev Neurosci. 1994; 16(3-4): 196-206.

80. Hassinger TD, Atkinson PB, Strecker GJ, Whalen L, Dudek FE, Kossel AH, et al. Evidence for glutamatemediated activation of hippocampal neurons by glial calcium waves. J Neurobiol. 1995; 28(2): 159-70.

81. Khodaie B, Lotfinia A, Ahmadi M, Lotfinia M, Jafarian M, Karimzadeh F, et al. Structural and functional effects of social isolation on the hippocampus of rats with traumatic brain injury. Behav Brain Res. 2015; 278: 55-65.

82. Lauritzen M, Hansen AJ. The effect of glutamate receptor blockade on anoxic depolarization and cortical spreading depression. J Cereb Blood Flow Metab. 1992; 12(2): 223-9.

83. Gill R, Andine P, Hillered L, Persson L, Hagberg H. The effect of MK-801 on cortical spreading depression 


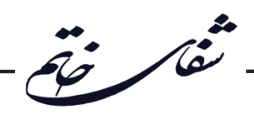

in the penumbral zone following focal ischaemia in the rat. J Cereb Blood Flow Metab. 1992; 12(3): 371-9.

84. Dermietzel R, Traub O, Hwang T, Beyer E, Bennett M, Spray D, et al. Differential expression of three gap junction proteins in developing and mature brain tissues. Proc Natl Acad Sci USA. 1989; 86(24): 10148-52.

85. Naus CC, Bechberger JF, Zhang Y, Venance L, Yamasaki H, Juneja SC, et al. Altered gap junctional communication, intercellular signaling, and growth in cultured astrocytes deficient in connexin43. J Neurosci Res. 1997; 49(5): 528-40.

86. Somjen G, Aitken P, Czeh G, Herrearas O, Jing J,
Young J. Mechanisms of spreading depression: a review of recent findings and a hypothesis. Can J Physiol Pharmacol. 1992; 70(S1): S248-S54.

87. Orkand R, Nicholls J, Kuffler S. Effect of nerve impulses on the membrane potential of glial cells in the central nervous system of amphibia. J Neurophysiol. 1966; 29(4): 788-806.

88. Hansson E, Muyderman H, Leonova J, Allansson L, Sinclair J, Blomstrand F, et al. Astroglia and glutamate in physiology and pathology: aspects on glutamate transport, glutamate-induced cell swelling and gap-junction communication. Neurochem Int. 2000; 37(2): 317-29. 